\title{
Influence of the Fictitious Grid on Flicker Assessment of Grid Connected Wind Turbine
}

\author{
K. Redondo, A. Lazkano, P. Saiz, J.J. Gutierrez, I. Azcarate and L.A. Leturiondo \\ Department of Communications Engineering \\ Faculty of Engineering, University of the Basque Country UPV/EHU \\ Alameda Urquijo, s/n - 48013 Bilbao (Spain) \\ Phone number: +34 94601 3901, e-mail: koldo.redondo@ehu.es
}

\begin{abstract}
This work studies the influence of the signal processing method on the flicker measurement of grid connected wind turbine in continuous operation according to the procedure detailed in the IEC 61400-21 standard. Two different methods for solving the fictitious grid are analyzed: Zero Crossing Detection and Short Time Fourier Transform. The deviations in the flicker measurement results obtained by applying both methods to actual signals from a measurement campaign are discussed. And the results are compared taking into account the grid impedance angle and the wind condition.
\end{abstract}

\section{Key words}

Power Quality, Wind Turbines, Flicker.

\section{Introduction}

Regarding Power Quality issues, Wind Turbines (WT) produce disturbances which are injected to the grid: voltage fluctuations or flicker, harmonics and interharmonics, and power changes (active and reactive power) [1]. These disturbances are due to the technical features of the WT, the meteorological conditions and the interaction between the WT and the grid.

Flicker emission is one of the main issues concerning the power quality of WT. Flicker is defined as the annoyance in the visual perception of a person who is subjected to light fluctuations produced by changes in the supply voltage.

The main cause of the flicker emission of WT is the power fluctuations produced by the WT itself. Power oscillations are due to switching operations (cut-in, cut-out and switch between generators), power control strategy and, mainly, environmental features (wind speed changes, wind shear and tower shadow) [2]-[4].

As reported in [5] the flicker emission is closely related to the features of the grid where the WT is connected. The influence of the grid has been described with two parameters: the grid impedance angle and the ratio between the short-circuit apparent power of the grid and the rated apparent power of the WT. About first parameter, for low grid impedance angles the dominant contribution to flicker emission is the active power fluctuation. While for high grid impedance angles it is reactive power fluctuation. And taking account power ratio, the flicker emission is bigger in weak grids than in strong grids [6]-[9].

The international standard for the assessment of the power quality characteristics of grid connected WT is IEC 61400-21 [10]. This standard establishes the power quality characteristics and the procedure for measuring and assessing them. Measurement procedures are specified to characterize the WT independently of the grid voltage fluctuations at the measurement site by using the circuit model of the WT-grid connection known as "fictitious grid".

This paper studies the influence of the signal processing method on flicker assessment, when applying the standard [10]. We have carried out the measuring campaign on a $225 \mathrm{~kW}$ WT in continuous operation. The study is focused on the differences between flicker measurements obtained with different signal processing methods used for solving the fictitious grid. The results are compared in terms of the grid impedance and the wind condition.

The paper is organized as follows: Section 2 describes the flicker assessment procedure stated in [10], and points out the relevance of an adequate selection of the signal processing methods for the implementation of the fictitious grid; Section 3 describes the characteristics of the WT and the data acquisition equipment used in the measurement campaign; Section 4 presents the results of flicker measurement and discusses the discrepancies between both signal processing methods. Section 5 stated the main conclusions. 


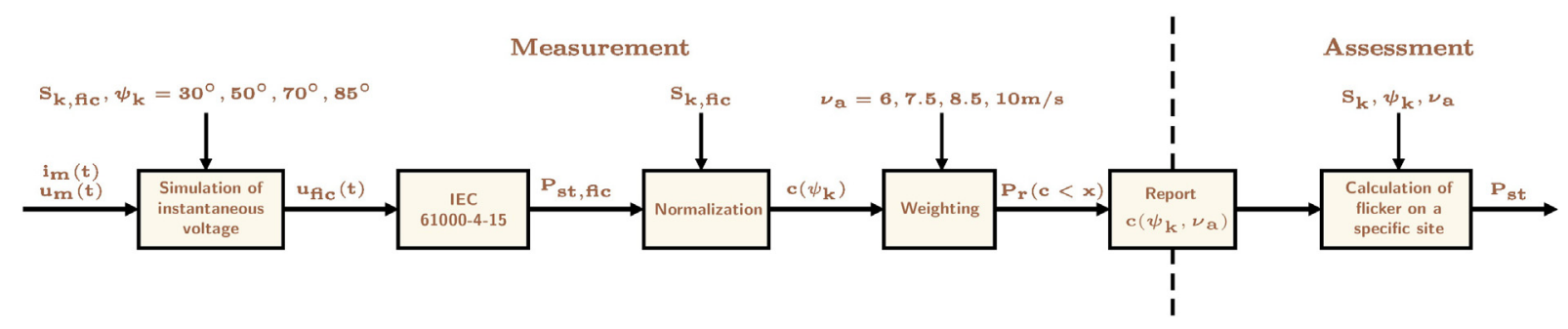

Fig. 1. Measurement and assessment procedures for flicker during continuous operation of the wind turbines in accordance with IEC 61400-21 standard [10].

\section{Flicker Measurement in Wind Turbines}

The IEC 61400-21 standard specifies a flicker assessment method that uses current and voltage time-series measured at the WT terminals, $i_{m}(t)$ and $u_{m}(t)$. Two different scenarios are defined for flicker measurement: continuous operation and switching operation. This work focuses on the continuous operation procedure according to Fig. 1.

The first block of Fig. 1 is known as "fictitious grid". For each pair of measured voltage and current 10-min timeseries, $u_{m}(t)$ and $i_{m}(t)$, the fictitious voltage $u_{f i c}(t)$ is computed. A proper fictitious short-circuit apparent power $S_{k, f i c}$ must be used and the calculation must be repeated for four grid impedance angles $\left(\psi_{k}=30^{\circ}, 50^{\circ}, 70^{\circ}\right.$ and $\left.85^{\circ}\right)$. The aim is to obtain the voltage fluctuations exclusively produced by the WT, and to achieve results which are independent of the grid condition at the test site. A more detail description of the fictitious grid will be described it in the subsequence section.

The second block of Fig. 1 processes the fictitious voltage $u_{f i c}(t)$ as the input to the IEC flickermeter. The IEC 610004-15 [11] standard provides a detailed description of the structure and functional specifications of a flickermeter. It establishes the short-term flicker severity $\left(P_{s t}\right)$ as the fundamental parameter to evaluate the annoyance. In this way a flicker emission value, $P_{s t, f i c}$, is obtained for each 10-min period of $u_{f i c}(t)$.

In the third block of Fig. 1 the flicker coefficient $c\left(\psi_{k}\right)$ is calculated for each 10 -min period, by normalizing $P_{\text {st fic }}$ value according to:

$$
c\left(\psi_{k}\right)=P_{s t, f i c} \cdot \frac{S_{k, f i c}}{S_{n}}
$$

where $S_{k, f i c}$ is the short-circuit apparent power of the fictitious grid and $S_{n}$ is the rated apparent power of the WT. The short-circuit ratio is suggested to be between 20 and 50 in [10], and is proposed to be a fixed value in [8].

In the fourth block of Fig. 1, a weighting factor is applied to each flicker coefficient $c\left(\psi_{k}\right)$, in order to scale the measured frequency of occurrence of the flicker coefficient to correspond with the wind speed distribution. A Rayleigh distribution is assumed, with four annual averages of the wind speed $\left(v_{a}=6,7.5,8.5\right.$ and $\left.10 \mathrm{~m} / \mathrm{s}\right)$.
Finally, the reported flicker coefficient $c\left(\psi_{k}, v_{a}\right)$ is obtained for different grid impedance angles $\left(\psi_{k}=30^{\circ}\right.$, $50^{\circ}, 70^{\circ}$ and $85^{\circ}$ ) and the aforementioned wind speeds $v_{a}$. The flicker coefficient $c\left(\psi_{k}, v_{a}\right)$ is calculated as the 99th percentile of the weighted accumulated distribution of the flicker coefficient values $c\left(\psi_{k}\right)$.

The flicker emission of a WT can be estimated with these values of the flicker coefficient $c\left(\psi_{k}, v_{a}\right)$. For a site where the grid impedance angle is $\psi_{k}$ and the annual wind speed average is $v_{a}$, the continuous operation flicker emission is estimated using:

$$
P_{s t}=c\left(\psi_{k}, v_{a}\right) \cdot \frac{S_{n}}{S_{k}}
$$

where $S_{n}$ is the rated apparent power of the WT and $S_{k}$ is the actual short-circuit apparent power at the point of common coupling (PCC).

The flicker coefficient for the actual $\psi_{k}$ and $v_{a}$ at the site can be obtained using a linear interpolation between the reported $c\left(\psi_{k}, v_{a}\right)$ values.

\section{A. Fictitious grid}

The first stage in the flicker processing procedure is to obtain a fictitious voltage $u_{f i c}(t)$ which characterizes the fluctuations caused exclusively by the WT. To that end, the standard defines the fictitious grid shown in Fig. 2. The WT is represented by a current generator, $i_{m}(t)$, which is the instantaneous measured line current. The grid is represented by an ideal phase-to-neutral voltage source, $u_{0}(t)$, series connected to the grid impedance modeled by a resistance $R_{f i c}$ in series with an inductance $L_{\text {fic }}$.

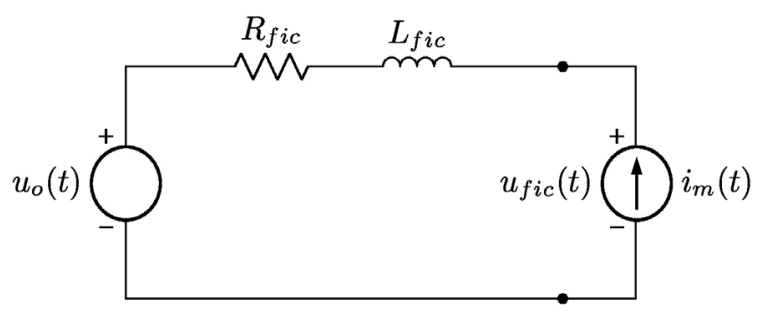

Fig. 2. Fictitious grid used to define $u_{f i c}(t)$ for flicker measurements in wind turbines. 
The fictitious grid gives a simulated voltage $u_{f i c}(t)$ according to:

$$
u_{f i c}(t)=u_{0}(t)+R_{f i c} \cdot i_{m}(t)+L_{f i c} \cdot \frac{d i_{m}(t)}{d t}
$$

The most sensitive aspect when solving (3) is focused on the signal $u_{0}(t)$. The ideal voltage source $u_{0}(t)$ must be constructed following two conditions. The first condition is that $u_{0}(t)$ must not have fluctuations, i.e. the flicker of $u_{0}(t)$ must be zero. And the second condition is that the electrical angle of $u_{0}(t)$ must be the same as that of the fundamental component of the measured voltage $u_{m}(t)$. Therefore $u_{0}(t)$ is defined as:

$$
u_{0}(t)=\sqrt{\frac{2}{3}} \cdot U_{n} \cdot \sin \left(\alpha_{m}(t)\right)
$$

where $U_{n}$ is the r.m.s. value of the nominal voltage of the grid, and $\alpha_{m}(t)$ is the electrical angle of the fundamental component of the measured voltage $u_{m}(t)$, that may be described as:

$$
\alpha_{m}(t)=2 \cdot \pi \cdot \int_{0}^{t} f(t) d t+\alpha_{0}
$$

where $f(t)$ is the frequency (that may vary over time) and $\alpha_{0}$ is the electrical angle at $t=0$.

The standard IEC 61400-21 does not specify which method must be used to generate $u_{0}(t)$. Nevertheless the selection of the proper signal processing technique could become an error source. Slight deviations in the estimation of $\alpha_{m}(t)$, produce negligible changes in the waveform of the $u_{f i c}(t)$ signal. However, those slight deviation substantially affect the $P_{s t, f i c}$ value [12].

\section{B. $\quad$ Proposed methods}

Two methods have been implemented for estimating the $u_{0}(t)$ signal: the Short Time Fourier Transform (STFT), and the classical Zero Crossing Detection (ZCD).

1) Short Time Fourier Transform (STFT): The STFT is typically applied to estimate the spectral components of a signal portion which change over the time. The input signal is divided in fragments of fixed time, known as windows. The Fourier Transform is applied to each window. For each complex result obtained, module and phase are extracted for each frequency. Window length is an important parameter concerning computational load and spectral resolution. The minimum window length necessary to characterize the fundamental component is one cycle. To avoid discontinuities in the estimation of the signal, a one cycle rectangular window with a sliding of one sample has been chosen. As only the fundamental component needs to be estimated, Goertzel algorithm is used. The Goertzel algorithm is a linear filtering technique that allows us to exclusively get the required component [13].

2) Zero Crossing Detection (ZCD): The estimation of the frequency of power systems using the zero-crossing technique is well known [14]. Given that we are detecting the $\pi$ radian instant of each cycle of $u_{m}(t)$, constructing the instantaneous phase of the signal $u_{m}(t)$ is straightforward. Working in the discrete domain, the algorithm searches for the positions of contiguous samples of $u_{m}(t)$ that mark a transition of values from positive to negative. To achieve a more precise approximation to the zero-crossing point, a linear interpolation between the points of the transition is used. Knowing the number and the fraction of the samples that make up a period, reconstruction of the instantaneous phase of the fundamental component is achieved, sharing the $2 \pi$ radians uniformly for each sampling instant.

\section{Measurement campaign}

In order to get a recorded time series database necessary for the study, we used a recording system developed by us [12].

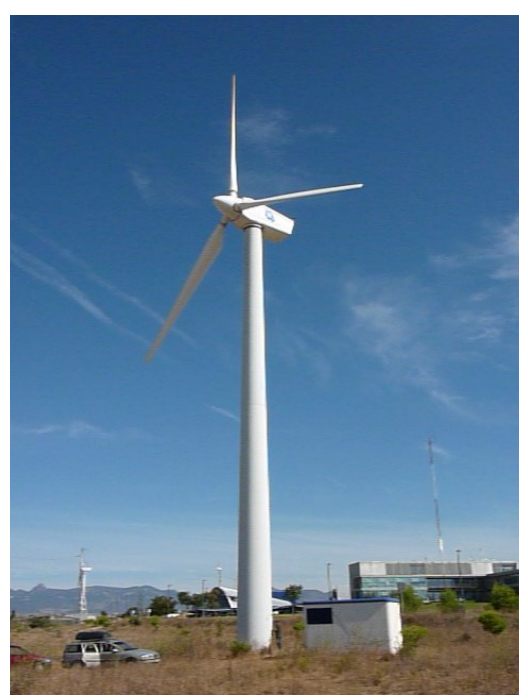

Fig. 3. Monitored wind turbine VESTAS V29 at Walqa Technological Park.

The recording system consists of two modules: a signal conditioning subsystem; and A/D conversion and storage subsystems.

The signal conditioning subsystem provides four voltage channels (available scales: 60, 150, 300 and $600 V_{r m s}$ ), four current channels (available scales: 50 and $500 A_{r m s}$ ) and two additional wind speed channels.

The conditioned signals are sampled using a sampling frequency of $25600 \mathrm{~S} / \mathrm{s}$, with the USB 6281 card from National Instruments (18-bits resolution). The acquisition 
is controlled by software developed in Matlab, which stores the acquired data on a hard disk.

The measuring campaign was made in Walqa Technological Park, Huesca, in the northeast of Spain in 2011 during three months. The analyzed turbine, VESTAS V29 shown in Fig. 3, is equipped with asynchronous generator directly connected to the grid, with pitchregulated power control. It has two different fixed speeds depending on the number of connected poles $(225 \mathrm{~kW} / 6$ poles or $50 \mathrm{~kW} / 8$ poles).

Three voltages, three currents and the wind speed signal were recorder creating a valuable database of signals for further analysis. Measurements were made at the connection point of the WT to the grid, at a rated voltage of $690 \mathrm{~V}$. The wind and power conditions recorded are shown in Fig. 4, where a scatter plot shows 10 min mean values of the electric power as a function of the wind speed.

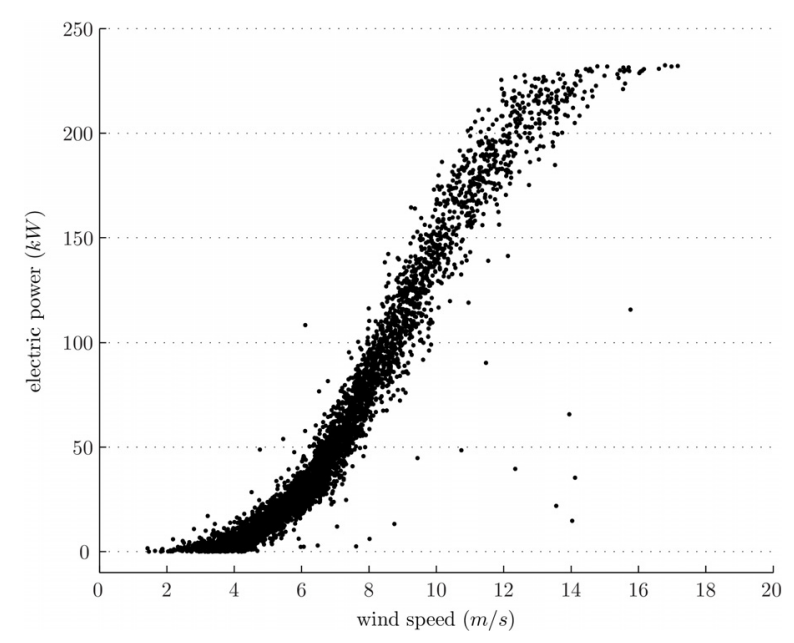

Fig. 4. Power performance curve of the VESTAS V29 $225 \mathrm{~kW}$ WT.

One week with significant wind speed values, between 4 and $11 \mathrm{~m} / \mathrm{s}$, has been selected. Periods of 10-min are prefiltered removing those containing cut-in, cut-out wind speeds or switching operations. Fig. 5 presents a histogram of the 10 -min periods analyzed.

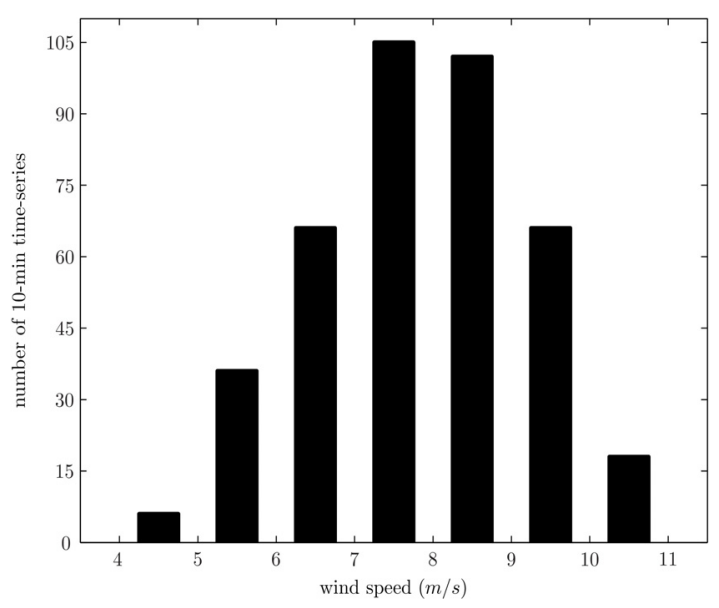

Fig. 5. 10-min time-series per wind speed bins.

\section{Results and discussion}

A comparison of the two signal processing methods proposed in Section 2 is described below. For voltage and current processing purposes, WT and fictitious grid parameters are specified in Table I.

Table I. - Flicker measurement parameters

\begin{tabular}{|c|c|c|}
\hline PARAMETER & DESCRIPTION & VALUE \\
\hline$U_{n}$ & $\begin{array}{c}\text { Nominal voltage of } \\
\text { the wind turbine }\end{array}$ & $690 \mathrm{~V}$ \\
\hline$I_{n}$ & $\begin{array}{c}\text { Rated current of } \\
\text { the wind turbine }\end{array}$ & $390 \mathrm{~A}$ \\
\hline$f_{0}$ & $\begin{array}{c}\text { Fundamental } \\
\text { grid frequency }\end{array}$ & $50 \mathrm{~Hz}$ \\
\hline$S_{k, f i c} / S_{n}$ & $\begin{array}{c}\text { Ratio between } \\
\text { power parameters }\end{array}$ & 30 \\
\hline$\psi_{k}$ & $\begin{array}{c}\text { Grid } \\
\text { impedance angle }\end{array}$ & $\begin{array}{c}30^{\circ}, 50^{\circ}, \\
70^{\circ} \text { and } 85^{\circ}\end{array}$ \\
\hline
\end{tabular}

Fig. $6 a$ and $6 \mathrm{~b}$ show the results achieved for STFT method and ZCD method respectively. The figures show the flicker coefficient $c\left(\psi_{k}\right)$ values calculated for each 10min time series of $u_{m}(t)$ and $i_{m}(t)$. The performance of the flicker coefficient $c\left(\psi_{k}\right)$ value related to the grid impedance angles is coherent with previous studies [5] and [8]. The flicker emission is correlated with the wind speed, higher wind speed result in higher flicker emission.

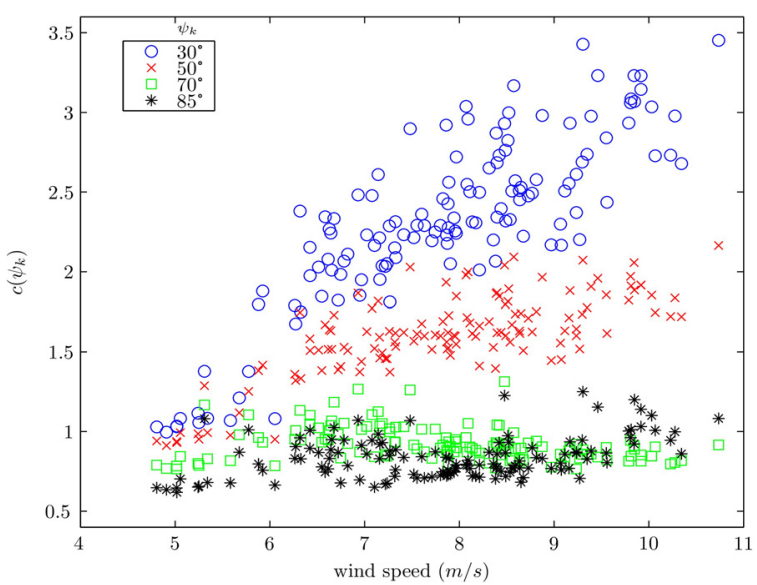

(a)

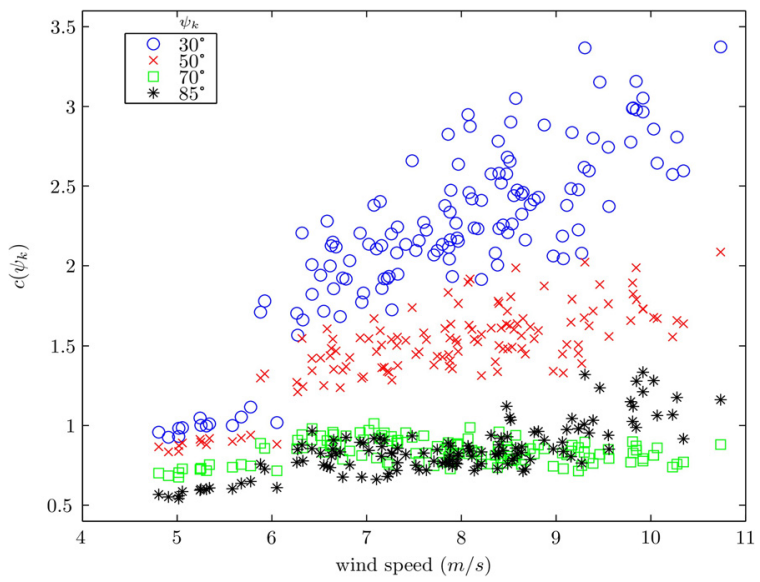

(b)

Fig. 6. Flicker coefficient $c\left(\psi_{k}\right)$ value calculated with: (a) STFT method, (b) ZCD method. 


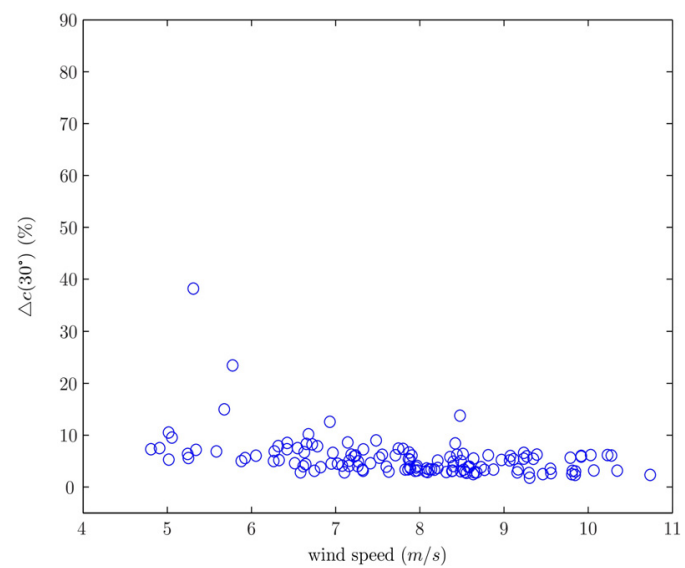

Fig. 7. Percentage change from ZCD method to STFT method in $c\left(30^{\circ}\right)$.

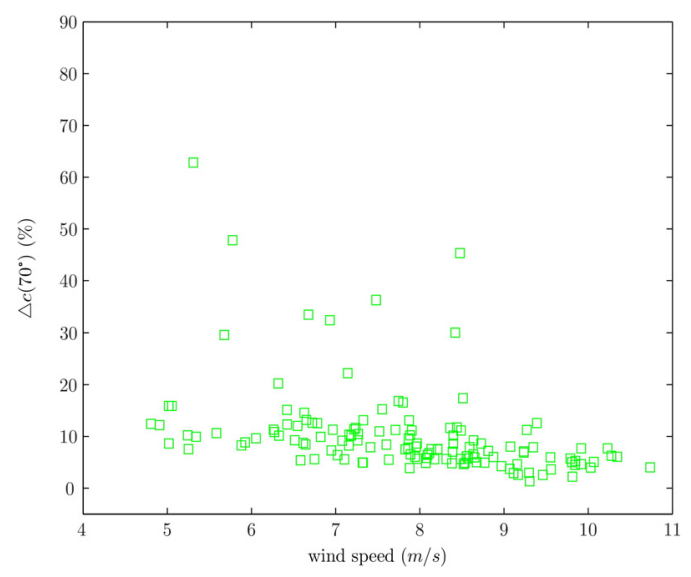

Fig. 9. Percentage change from ZCD method to STFT method in $c\left(70^{\circ}\right)$.

In order to investigate the differences between the two processing methods, Fig. 7 to Fig. 10 show the percentage differences of the flicker coefficient $c\left(\psi_{k}\right)$ values for both methods. In particular, the percentage change from flicker coefficient $c\left(\psi_{k}\right)$ calculated with ZCD to flicker coefficient $c\left(\psi_{k}\right)$ calculated with STFT is computed according to:

$$
\Delta c\left(\psi_{k}\right)=100 \cdot \frac{c_{S T F T}\left(\psi_{k}\right)-c_{Z C D}\left(\psi_{k}\right)}{c_{Z C D}\left(\psi_{k}\right)}
$$

Figures 7 to 9 show that STFT method overestimates the flicker emission with respect to ZCD method. The differences can be significant (more than 20\%), although the mean value of the difference is around $10 \%$ (see Table II).

Table II. - Mean values of the percentage changes of flicker coefficient $c\left(\psi_{k}\right)$.

\begin{tabular}{|c|c|}
\hline $\begin{array}{c}\text { IMPEDANCE } \\
\text { ANGLE }\end{array}$ & $\begin{array}{c}\text { MEAN VALUE OF } \\
\text { PERCENTAGE CHANGE }\end{array}$ \\
\hline $30^{\circ}$ & $5.5 \%$ \\
\hline $50^{\circ}$ & $8.2 \%$ \\
\hline $70^{\circ}$ & $10.3 \%$ \\
\hline $85^{\circ}$ & $7.8 \%$ \\
\hline
\end{tabular}

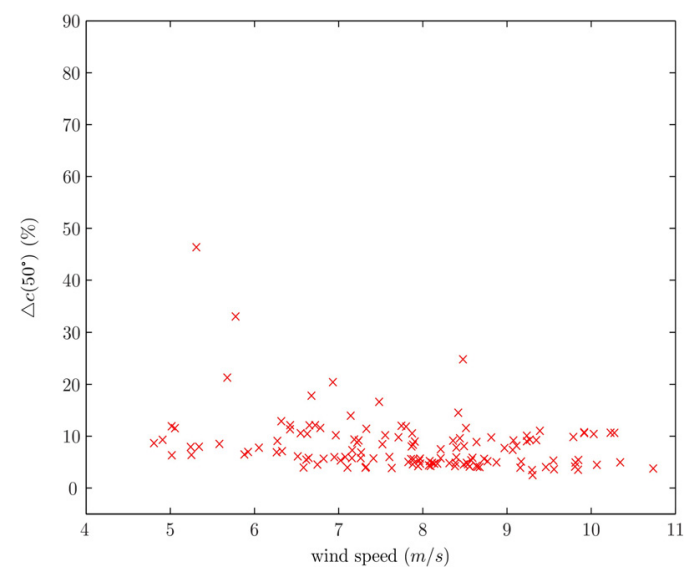

Fig. 8. Percentage change from ZCD method to STFT method in $c\left(50^{\circ}\right)$.

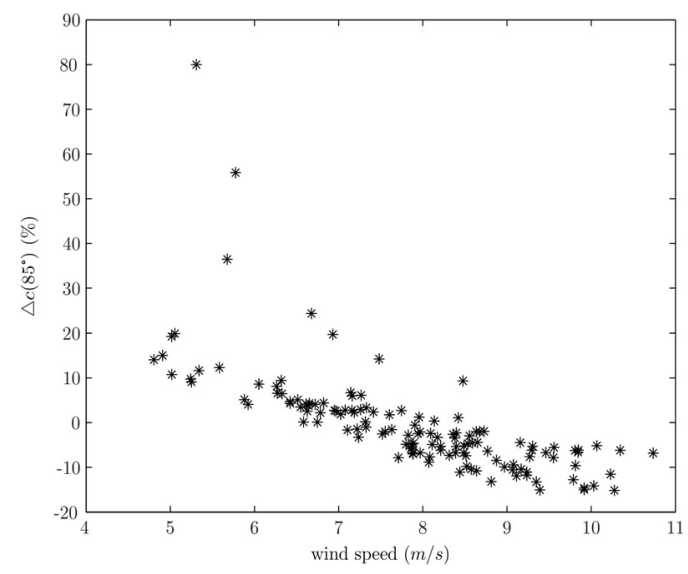

Fig. 10. Percentage change from ZCD method to STFT method in $c\left(85^{\circ}\right)$.

Behavior is different in Fig. 10. The figure shows a decreasing trend of the percentage change, from overestimation to underestimation with increasing wind speed.

Moreover, the efficiency of the method in terms of the computational load is an important feature. STFT requires greater computational load than ZCD. According to Table III, STFT requires close to three times more multiplications and 30\% more sums than ZCD. The table shows the numbers of operations per one second duration of signal, which has a $25600 \mathrm{~Hz}$ sampling rate.

Table III. - Computational load.

\begin{tabular}{|c|r|c|}
\hline \multirow{2}{*}{ METHOD } & $\begin{array}{c}\text { TYPE OF } \\
\text { OPERATIONS }\end{array}$ & $\begin{array}{c}\text { OPERATIONS } \\
\text { PER SECOND }\end{array}$ \\
\hline \multirow{2}{*}{ STFT } & sums & 204.795 \\
\cline { 2 - 3 } & multiplications & 204.796 \\
\hline \multirow{2}{*}{ ZCD } & sums & 153.650 \\
\cline { 2 - 3 } & multiplications & 76.800 \\
\hline
\end{tabular}




\section{Conclusions}

The main conclusion of this work is that the processing method used for solving the fictitious grid can give rise to significant differences in the flicker emission value assessed.

The analysis has been performed using actual data. The measurement campaign was made on a $225 \mathrm{~kW}$ wind turbine, creating a valuable database of signals for this study and for further analysis.

During the last ten years doubts about the accuracy of different implementations of the IEC flickermeter have been reported in written form, and the standard [11] has been reedited in 2010 including compliance tests. Doubts about the accuracy of the flicker characterization of WT have been only reported orally. Utilities in the regulated market were not worried about this issue because low flicker values obtained. But, as the installed wind capacity is expected to increase significantly in the next ten years, global emission limits of flicker could be compromised [15].

Based on the above detailed discussion this paper suggests that the processing methods should be stated in the report of power quality assessment of grid connected wind turbines. Moreover, it would be interesting to design compliance test with measurement requirements in [10] about flicker. In this way, the inter-comparability of the measurements would be strengthened, and the measurement systems would become more coherent when applied to the same input signals.

\section{Acknowledgement}

The authors would like to thank Walqa Technological Park in Huesca (Spain) for making the voltage and current signals of wind turbine available free of charge for the purpose of this work.

This work was supported by Government of Basque Country through the PhD studentship BFI-2012-315, and the University of the Basque Country UPV/EHU through the project UFI11/16 and the $\mathrm{PhD}$ studentship PIF2011/169.

\section{References}

[1] T. Ackermann, "Wind Power in Power Systems", John Wiley \& Sons, 2005.

[2] A. Larsson, "Flicker emission of wind turbines during continuous operation". IEEE Transactions on Energy Conversion. 2002. pp. 114-118.

[3] A. Larsson, "Flicker emission of wind turbines caused by switching operations". IEEE Transactions on Energy Conversion. 2002. pp. 119-123.

[4] A. Lazkano, K. Redondo, P. Saiz, J.J. Gutierrez, I. Azcarate, L.A. Leturiondo and J. Barros, "Case study: Flicker emission and $3 P$ power oscillations on fixed-speed wind turbines". IEEE 15th International Conference on Harmonics and Quality of Power (ICHQP), June 2012. pp. 268-273.
[5] P. Sørensen, J.O. Tande, L.M. Søndergaard and J.D. Kledal, "Flicker Emission Levels from Wind Turbines". Wind Engineering. Vol. 20 no. 1. 1996.

[6] J. Schoene, T.E. McDermott, C. Smith, R. Zavadil and J. Lamoree, "Flicker from Distributed Wind Generation". 2011 IEEE Power and Energy Society General Meeting, July 2011. pp.1-9.

[7] T. Thiringer, T. Petru, and S. Lundberg, "Flicker Contribution From Wind Turbine Installations". IEEE Transactions on Energy Conversion, Vol. 19 no. 1. 2004. pp. 157-163.

[8] L.S. Christensen, P.E. Sørensen, T.S. Sørensen and H.K. Nielsen, "Evaluation of measuring methods for flicker emission from modern wind turbine". 2009.

[9] B. Barahona, P. Sørensen, L. Christensen, T. Sørensen, H.K. Nielsen and X.G. Larsén, "Validation of the Standard Method for Assessing Flicker From Wind Turbines". IEEE Transactions on Energy Conversion. Vol. 26 no. 1. March 2011. pp. 373-378.

[10] IEC-61400-21. International Electrotechnical Commission. "Wind Turbines - Part 21: Measurement and assessment of power quality characteristics of grid connected wind turbines". Edition 2. August 2008.

[11] IEC-61000-4-15. International Electrotechnical Commission. "Electromagnetic Compatibility (EMC) Part 4: Testing and Measurements Techniques - Section 15: Flickermeter - Functional and Design Specifications". Edition 2. 2010.

[12] J.J. Gutierrez, J. Ruiz, L.A. Leturiondo and A. Lazkano, "Flicker Measurement System for Wind Turbine Certification". IEEE Transactions on Instrumentation and Measurement. Vol. 57 no. 12. Dec 2008. pp. 375-382.

[13] J.G. Proakis and D.G. Manolakis. "Digital signal processing: principles, algorithms, and applications". Prentice-Hall International editions. 1996.

[14] J. Lee and M.J. Devaney. "Accurate measurement of line frequency in the presence of noise using time domain data". IMTC/94 Instrumentation and Measurement Technology Conference, May 1994. pp. 1016-1019.

[15] M. Ammar, "Flicker Emission of distributed wind power: A review of impacts, modeling, grid codes and mitigation techniques". 2012 IEEE Power and Energy Society General Meeting, July 2012. Montreal, Quebec, Canada. pp. 1-7. 